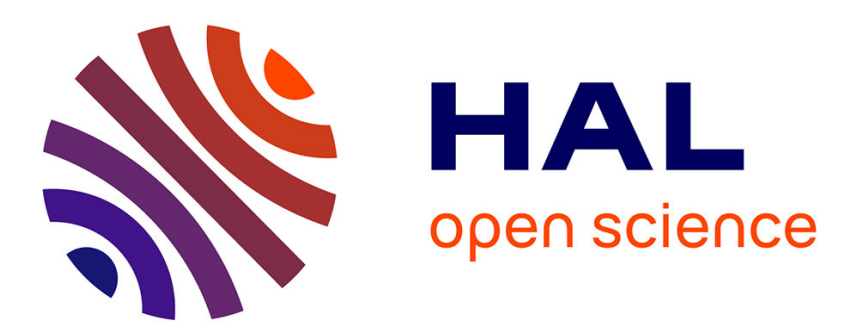

\title{
Approche énergétique du maillage adaptatif et des critères d'erreur
}

Dimitrios Ladas, Vincent Mazauric, Gérard Meunier, Olivier Chadebec

\section{To cite this version:}

Dimitrios Ladas, Vincent Mazauric, Gérard Meunier, Olivier Chadebec. Approche énergétique du maillage adaptatif et des critères d'erreur. Revue Internationale de Génie Electrique, 2008, 11 (23/2008), pp.173-183. hal-03048129

\section{HAL Id: hal-03048129 \\ https://hal.science/hal-03048129}

Submitted on 9 Dec 2020

HAL is a multi-disciplinary open access archive for the deposit and dissemination of scientific research documents, whether they are published or not. The documents may come from teaching and research institutions in France or abroad, or from public or private research centers.
L'archive ouverte pluridisciplinaire HAL, est destinée au dépôt et à la diffusion de documents scientifiques de niveau recherche, publiés ou non, émanant des établissements d'enseignement et de recherche français ou étrangers, des laboratoires publics ou privés. 


\title{
Approche énergétique du maillage adaptatif et des critères d'erreur
}

\author{
Dimitrios Ladas* — Vincent Mazauric* \\ Gérard Meunier** _ Olivier Chadebec** \\ * Schneider Electric, Innovation Direction \\ F-38050 Grenoble cedex 9 \\ dimitrios.ladas@schneider-electric.com \\ ** G2ELAB, INPG-UJF-CNRS UMR 5269 \\ ENSIEG - BP 46 \\ F-38402 Grenoble
}

RÉSUMÉ. Afin d'améliorer les modélisations éléments finis des courants de Foucault macroscopiques, nous proposons une approche originale de l'évaluation d'erreur pour le maillage adaptatif. Un critère d'erreur, fondé sur une conservation locale de la puissance, est présenté. Nous proposons quelques points de réflexion sur l'importance de l'ordre des éléments dans cette approche. Quelques résultats de remaillage $1 D$ sont présentés pour valider le critère d'erreur proposé.

ABSTRACT. In order to improve the Finite Element modelings of macroscopical eddy-currents, we propose an original approach of error evaluation for adaptive meshing. An error criterion, based on a local power conservation, is presented. We propose to focus on the importance of element order in this aproach. Some remeshing results for $1 D$ cases are presented to validate the error criterion proposed.

MOTS-CLÉS : courants de Foucault, critère d'erreur, maillage adaptatif, ordre des éléments.

KEYWORDS: eddy currents, error criterion, adaptive meshing, element order. 


\section{Introduction}

Afin de répondre aux objectifs d'optimisation de l'efficacité énergétique des dispositifs, il est nécessaire de disposer d'outils de modélisation à même d'évaluer précisément les différentes énergies, sous forme noble ou dégradée, mises en jeu au cours de la conversion électromécanique.

Les courants de Foucault macroscopiques sont induits par une excitation électrique variable et/ou le mouvement de pièces conductrices massives. Ces phénomènes dissipatifs amoindrissent les performances des actionneurs électromécaniques et leur nonprise en compte dans les phases de conception de produit amènent des disfonctionnements. Actuellement, ils sont correctement modélisés en éléments finis 2D, mais en 3D de nombreux problèmes subsistent, à cause, entre autre, du maillage. Le problème critique est lié à la prise en compte de l'effet pelliculaire, et à la nécessité de fournir un maillage adapté à ce phénomène physique. Nous proposons ainsi un critère d'erreur novateur adapté au remaillage des conducteurs massifs. Nous discuterons de l'importance de l'ordre des éléments dans cette approche, et nous proposons un exemple 1D en magnétoharmonique pour valider le critère.

\section{Modélisation éléments finis et maillage adaptatif}

\subsection{Cadre thermodynamique}

De récents travaux (Mazauric, 2003), (Mazauric, 2004) ont montré que les équations de Maxwell peuvent être déduites par une approche thermodynamique de l'électromagnétisme. L'équilibre magnétostatique est obtenu en écrivant la stationnarité de l'enthalpie libre par rapport aux variations potentielles du champ magnétique. L'équation de Maxwell-Faraday, qui régit les phénomènes dynamiques est déduite de la minimisation de la fonctionnelle [1]. Cette approche apporte une justification intrinsèque aux éléments finis pour traiter les problèmes d'électromagnétisme. En effet, cette méthode revient à construire une approximation des formulations variationnelles sur un maillage et de trouver le champ $\mathrm{H}$, dans un espace de fonctions approchées, qui vérifie le minimum de la fonctionnelle approchée.

$$
\wp_{\text {Fonctionnelle }}=\iiint_{\text {Conducteurs }}\left[\rho \mathbf{j}^{2}+\frac{\partial(\mathbf{B H})}{\partial t}\right] d v
$$

où $\rho$ est la résistivité électrique, $\mathbf{j}$ la densité de courant, $\mathbf{B}$ l'induction magnétique et H le champ magnétique.

Cette approche permet de recadrer les différents objectifs de modélisation autour d'une même problématique : l'énergie. La méthode des éléments finis apparaît naturelle pour traiter les problèmes de conversion d'énergie, car elle repose sur la minimisation de fonctionnelles énergétiques. Ainsi il était tentant d'étendre la démarche au maillage adaptatif. 


\subsection{Travaux précédents}

En magnétostatique, le maillage adaptatif a été traité dans de nombreux travaux. (Jänicke et al., 2004) propose une technique complète, depuis l'évaluation de l'erreur au remaillage, le critère d'erreur utilisé restant classique. Certains articles ont présenté des approches énergétiques :

- dans (Pinchuk et al., 1985), un critère d'erreur local qui évalue la précision du calcul de l'énergie stockée dans chaque élément est proposé;

- pour les cas de magnétostatique avec mouvement, (Dufour et al., 2001) propose de travailler sur la maximisation de la fonctionnelle de co-énergie en fonction de la position des nœuds.

- (Rikabi et al., 1988a) propose d'évaluer l'erreur sur la loi de comportement, à partir de la théorie des principes variationnels complémentaires, en utilisant le Ligurien.

D'autres articles s'intéressent à des techniques de maillage adaptatif dédiées aux problèmes avec courants de Foucault :

- (Rikabi et al., 1988b) étend les travaux en statique aux problèmes de courants induits, toujours avec l'utilisation des formulations complémentaires ;

- (Li et al., 1994) propose 2 estimateurs d'erreur, le premier fondé sur le Ligurien et l'autre sur une discontinuité numérique du champ;

- (Dufour et al., 2002) utilise une solution locale dans les régions conductrices pour bâtir un estimateur d'erreur;

- (Sterz et al., 2006) présente un estimateur d'erreur qui évalue la précison sur l'équation de Maxwell-Ampère.

En pratique, le maillage adaptatif n'est pas très répandu pour les régimes transitoires. Les critères utilisés se limitent à des considérations géométriques ou de continuité. Nous proposons ici un point de vue nouveau, dans lequel le critère d'erreur prend en compte la nécessité de précision sur les calcul des grandeurs énergétiques.

\subsection{Cas particulier des régimes dynamiques}

Une première approche nous a conduit à une recherche de critère d'erreur a priori. Il s'agissait d'étendre les travaux en magnétostatique de (Dufour et al., 2000) aux régimes variables. L'idée était de trouver un critère valable à la fois pour les régimes transitoire et harmonique. Pour résoudre l'équation qui régit les courants de Foucault [2] (en formulation $\mathrm{H}$ ) dans les régions conductrices, on stationnarise la fonctionnelle [1] par rapport aux variations de $\mathrm{H}$, en projection sur un maillage.

$$
\operatorname{rot} \rho \operatorname{rot} \mathbf{H}-\frac{\partial \mu \mathbf{H}}{\partial t}=0
$$

avec $\mu$ la perméabilité magnétique du conducteur. 
Le problème des courants induits en régime harmonique ne peut pas être transformé sous forme variationnelle (Bossavit, 1998). La solution est un point-selle obtenu en stationnarisant la fonctionnelle complexe [3] :

$$
\wp_{\text {Complexe }}=\iiint_{\text {Conducteurs }}\left[\rho \mathbf{j} \cdot \mathbf{j}^{*}+i \omega \mu \mathbf{H} \cdot \mathbf{H}^{*}\right] d v
$$

où $i$ phaseur complexe, $\omega$ la pulsation du champ d'excitation.

A cause de ce problème, les idées de minimisation en fonction du maillage ont été abandonnées au profit d'une approche par critère d'erreur a posteriori.

\section{Vers une nouvelle approche de l'évaluation de l'erreur}

\subsection{Critère d'erreur énergétique et critère classique}

L'évaluation d'erreur que nous proposons se fait élément par élément, et consiste à calculer l'écart entre la puissance rentrante dans un élément et la puissance contenue dans cet élément, qui doivent être égales si le maillage est «parfait ». La puissance contenue dans l'élément $\Omega_{i}$ est la somme des pertes joule et de la puissance magnétique :

$$
P_{\text {Contenue }}=\iiint_{\Omega_{i}}\left[\rho \mathbf{j}^{2}+\mathbf{H} \frac{\partial \mathbf{B}}{\partial t}\right] d v
$$

La puissance rentrante est obtenue grâce au flux du vecteur de Poynting à travers le contour $\Gamma_{i}$ de l'élément :

$$
P_{\text {Entrante }}=-\oint_{\Gamma_{i}}[\mathbf{E} \wedge \mathbf{H}] d s
$$

où $\mathbf{E}$ est le champ électrique.

Le nouveau critère d'erreur assurant la conservation locale de la puissance s'écrit donc :

$$
\begin{aligned}
\epsilon_{i} & =\left|P_{\text {Contenue }}-P_{\text {Entrante }}\right|_{\text {elt } i} \\
& =\left|\iiint_{\Omega_{i}}\left[\rho \mathbf{j}^{2}+\mathbf{H} \frac{\partial \mathbf{B}}{\partial t}\right] d v+\oint_{\Gamma_{i}}[\mathbf{E} \wedge \mathbf{H}] d s\right|
\end{aligned}
$$

Après quelques calculs, en utilisant le théorème Green-Ostogradsky, l'erreur s'écrit :

$$
\epsilon_{i}=\left|\iint_{\Omega_{i}}\left[\left(\operatorname{rot} \mathbf{E}+\frac{\partial \mathbf{B}}{\partial t}\right) \cdot \mathbf{H}-(\operatorname{rot} \mathbf{H}-\mathbf{j}) \cdot \mathbf{E}\right] d v\right|
$$


Ce critère est adapté au raffinement de maillage dans les zones conductrices. Il est évident que cette écriture générique [8] ne va pas donner les mêmes résultats suivant la formulation utilisée, mais le sens du critère reste le même :

- en $\mathbf{A}-\mathbf{V}$, le premier terme de [8] s'annule. Le critère évalue la précision sur l'équation de Maxwell-Ampère.Cette évaluation est proche des critères classiques, mais l'information énergétique donnée par le produit avec le champ électrique $\mathbf{E}$ apporte une précision supplémentaire;

- en $\mathbf{T}-\boldsymbol{\Phi}$, qui sont liées aux variables naturelles obtenues à partir de l'approche thermodynamique (Mazauric, 2003), le second terme de [8] s'annule. Cela donne un sens plus puissant au critère qui évalue alors la précision sur l'équation de MaxwellFaraday, qui régit les phénomènes induits. De plus le produit par le champ magnétique H s'adapte bien aux problèmes par courants de Foucault à cause de l'évanescence du champ.

Ce nouveau critère, robuste et suffisemment général est à même de piloter n'importe quel remailleur. Nous allons discuter maintenant d'un aspect intéressant qui concerne la nécessité de prendre en compte ou non les éléments voisins dans le calcul des erreurs.

\subsection{Importance de l'ordre des éléments}

Lorsqu' on s'intéresse au calcul de [5] ou [9], on a le choix entre :

- utiliser les valeurs des champs dans l'élément, et ainsi évaluer exactement la puissance « entrant » dans l'élément ;

- utiliser les valeurs des champs dans les éléments voisins, donc évaluer la puissance « sortant » des voisins.

Ce dernier calcul se rapproche d'un calcul de discontinuité numérique, entre grandeurs énergétiques certes, mais il reste assez semblable dans l'approche avec les travaux passés.

Le point de vue proposé dans cet article est d'utiliser les informations de l'élément lui-même, dans la mesure où il existe un couplage intrinsèque (lié à la matrice éléments finis) entre cet élément et ses voisins. Mais cette affirmation est vraie uniquement si l'approximation du champ dans l'élément est assez riche, autrement dit si l'ordre des éléments est suffisamment élevé.

Afin d'illustrer le propos, nous proposons le critère classique suivant :

$$
\epsilon \underset{\text { classique }}{i}=\oint_{\Gamma_{i}} \mathbf{B} d s
$$

qui revient à estimer la précision sur le calcul magnétostatique, en évaluant la conservation du flux magnétique. Ce critère est adapté aux formulations $\mathbf{T}-\Phi$. 


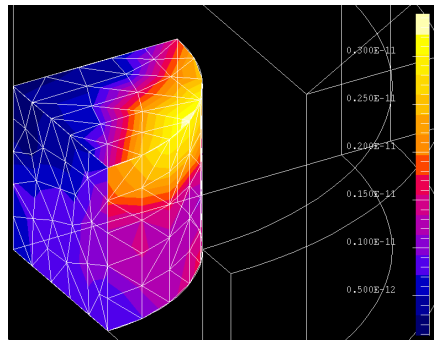

Critère énergétique [6]

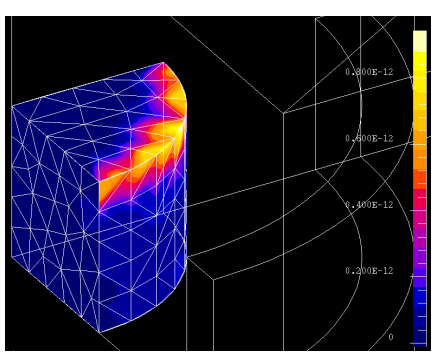

Ordre 1

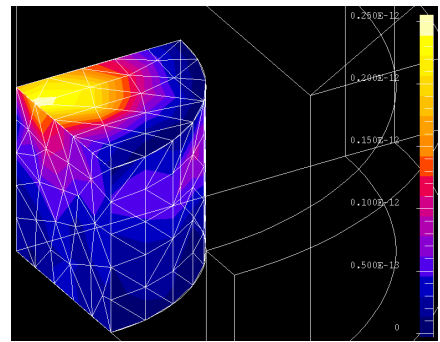

Critère classique [9]

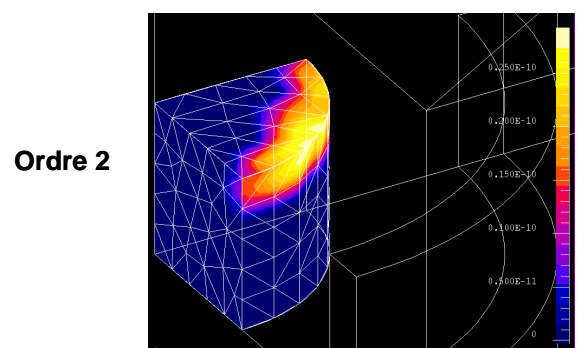

Figure 1. Erreur selon le critère énergétique [6] et selon le critère classique [9]. Les zones sensibles à la qualité du maillage sont situées vers les bords du cylindre et plus particulièrement sur l'arête. Alors que le critère énergétique [6] indique toujours les bonnes zones, le critère classique [9] est mis en défaut à l'ordre 1.

Nous allons présenter quelques résultats comparatifs, afin de voir les différentes implications de l'ordre des éléments pour les critères [6] et [9].

\subsection{Résultats sur l'ordre des éléments}

Nous présentons le cas du chauffage par induction où un noyau ferromagnétique est plongé dans un champ sinusoïdal créé par une bobine. Ce cas est modélisé sous le logiciel éléments finis Flux3D, qui utilise les formulations $\mathbf{T}-\Phi$. Tous les critères d'erreur sont calculés sans utilisation des éléments voisins.

La figure 1 montre les zones « à remailler » selon les critères et l'ordre des éléments. Ces résultats sont explicités avec le tableau 1. Nous soulignerons 2 points importants :

- Le $1^{\text {er }}$ ordre ne permet pas de disposer d'erreur par élément sans recourir aux éléments voisins pour le calcul. Cela se traduit par des aberrations avec le critère [9];

- Le critère énergétique [6] est égal à la puissance magnétique au $1^{\mathrm{er}}$ ordre. Ce n'est pas une erreur au sens stricte mais, on constate que les zones pointées sont cohérentes avec le $2^{\text {nd }}$ ordre. Cette évaluation n'est donc pas aberrante, et on retrouve ce 
type de considérations dans (Labridis, 2000). Nous utiliserons ce résultat pour le cas 1D proposé dans la suite, avec des éléments du $1^{\mathrm{er}}$ ordre.

\begin{tabular}{|c|c|c|}
\cline { 2 - 3 } \multicolumn{1}{c|}{} & Critère énergétique & Critère classique \\
\hline Ordre 1 & $\epsilon_{i}=\iiint_{\Omega_{i}}\left[\mathbf{H} \frac{\partial \mathbf{B}}{\partial t}\right] d v$ & $\begin{array}{c}\epsilon_{i} \simeq 0 \\
\text { (pointe des zones où } \\
\text { le raffinement est inutile) }\end{array}$ \\
\hline Ordre 2 & Erreur pertinente & Erreur pertinente \\
\hline
\end{tabular}

Tableau 1. Signification de l'erreur pour les 2 critères selon l'ordre des éléments, en n'utilisant pas les voisins pour les calculs. A l'ordre 1, seul le critère énergétique conserve un sens. En effet, le flux du vecteur de Poynting étant exactement égal aux pertes joule, le critère est égal à la puissance magnétique. A l'ordre 2, les 2 critères rendent compte des zones à affiner

\section{Validation du critère sur un cas 1D simple avec le remaillage d'un conducteur}

Ce cas test a pour but de valider le critère d'erreur proposé sur un cas simple. Nous avons utilisé la plaque symétrique 1D excitée par un champ extérieur sinusoïdal. La solution analytique étant connue, elle permettait d'évaluer la précision dans les calculs de puissance pour différents maillages. Le remaillage a été testé avec 2 techniques :

- par déplacement de nœuds ;

- par rajout de nœuds.

\subsection{Déplacement de nœuds}

Nous avons exploré une problématique qui consiste à essayer d'obtenir le meilleur maillage à nombre de nœuds donnés. La position des nœuds est optimisée sous une contrainte d'espace (les nœuds ne doivent pas sortir du domaine) avec une fonction objectif qui rend compte de la meilleure conservation locale de puisssance, en accord avec le critère [6]. Toutefois, cette approche nécessitant la prise en compte du déplacement de tous les nœuds, nous avons dû adapter le critère, qui se prête plus à une approche de remaillage par ajout de nœuds. Nous avons utilisé un algorithme génétique : différentes configurations de nœuds ont été comparées, en analogie avec les théories de l'évolution, et à chaque pas, la meilleure configuration est gardée. La fonction objectif devait prendre en compte la variance de l'erreur locale par rapport à la valeur moyenne. L'optimisation a donc consisté à évaluer :

$$
\min \left[\sum_{\text {Elements }}\left|\epsilon_{i}-\left\langle\epsilon_{i}\right\rangle\right|\right]
$$

où $\epsilon$ représente l'erreur relative et \langle\rangle l'opérateur moyenne spatiale. 


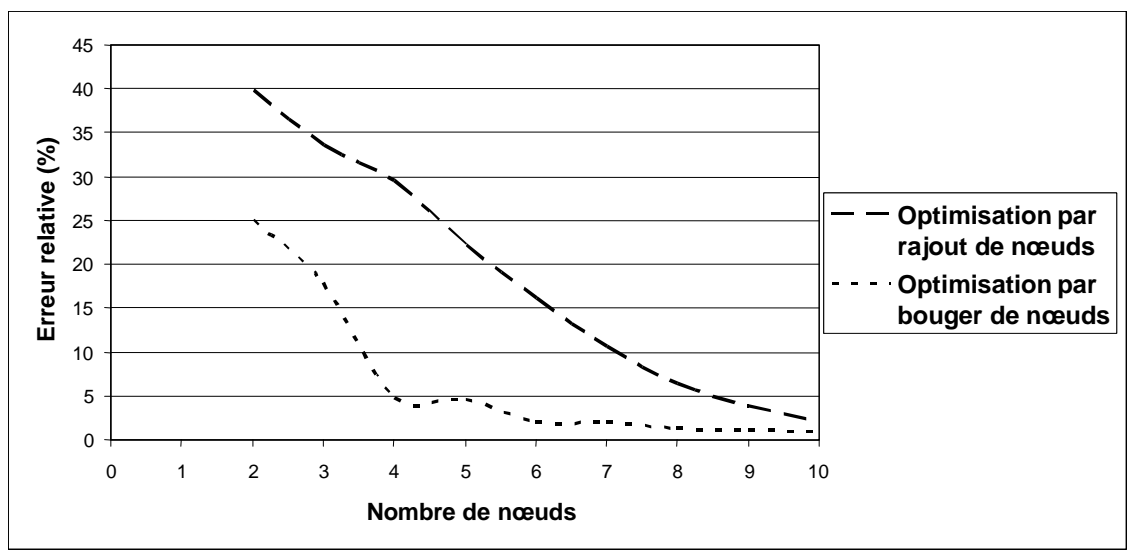

Figure 2. Erreur relative sur la puissance totale par rapport à la valeur analytique en fonction du nombre de nouds, pour les 2 techniques de remaillage. Même si la technique par bouger de nouds est plus efficace (les oscillations sont dues à des problèmes de convergence liés à l'algorithme génétique utilisé), le coût en temps de calcul est tel qu'il devient impossible de l'étendre à des cas plus compliqués

Ainsi le maillage optimisé présente la même erreur sur chaque élément. En plus, nous vérifions que les différentes puissances convergent vers les valeurs analytiques (figure 2). L'inconvénient de cette technique est son coût en temps de calcul. En 1D, la méthode est séduisante, mais même sur un cas 2D simple, il est difficile de la mettre en œuvre.

\subsection{Ajout de nouds}

Cette technique est classique, et a souvent été utilisée pour le maillage adaptatif. L'erreur est évaluée sur chaque élément du maillage (critère [6]) et on ajoute un nœud dans l'élément où l'erreur est la plus forte. On itère cette procédure jusqu'à obtenir une bonne convergence sur une grandeur globale. Nous avons choisi, comme critère d'arrêt, la précision sur le calcul de la puissance totale. La figure 3 montre la diminution de l'erreur locale entre le maillage initial à 2 nœuds et la configuration optimisée à 8 nœuds. L'erreur absolue sur chaque élément diminue et la puissance totale converge (figure 2). La figure 4 montre l'amélioration du profil du champ magnétique. Cette technique est comparée à la technique par bouger de nœuds (figure 2), en comparant les résultats sur la précision des calculs de puissance totale. La technique par bouger de nœuds apparaît plus précise car elle n'est pas tributaire du maillage initial. 

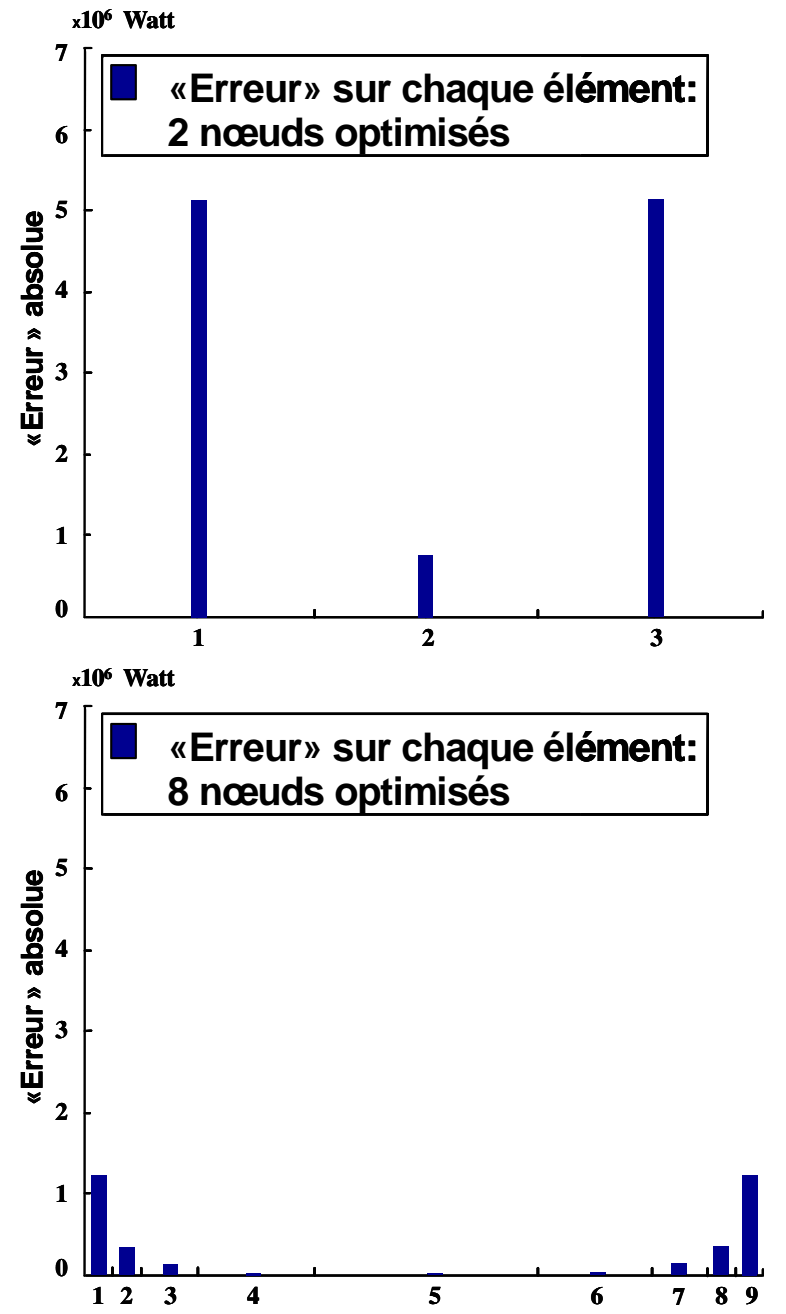

Figure 3. «Erreur » sur chaque élément pour le maillage initial à 2 nouds et pour la configuration optimisée par rajout de næuds. A cause des éléments du premier ordre, cette erreur est égale à la puissance magnétique, mais on constate que ce critère pointe toujours les zones pertinentes à remailler 

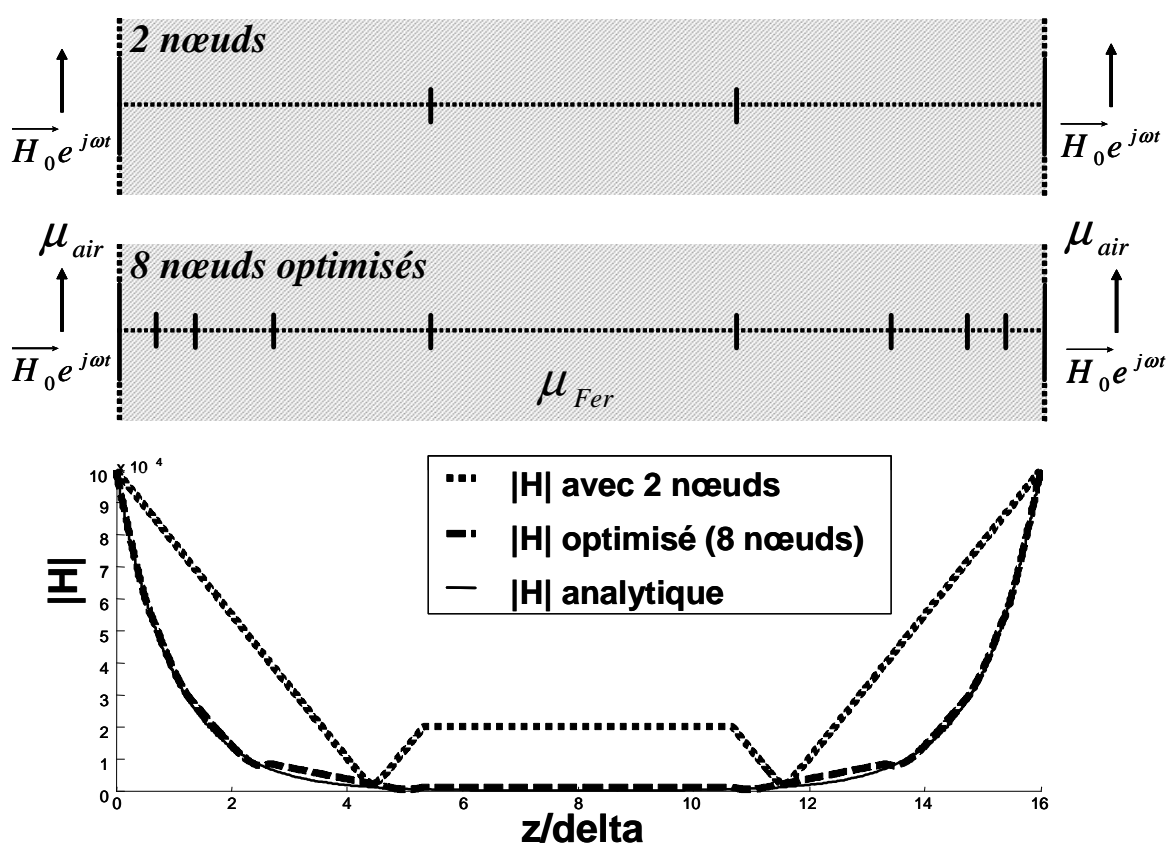

Figure 4. Module de $\mathbf{H}$ dans le conducteur pour 2 nœuds équirépartis, pour 8 nœuds optimisés par rajout de nœuds sur le maillage initial à 2 nœuds, et pour la solution analytique. La position des nouds est représentée. La méthode permet de diminuer de façon notable les aberrations sur le profil du champ, dues aux éléments du $1^{\text {er }}$ ordre. En effet, la valeur du champ magnétique aux nouds est complexe et le module du champ dans le conducteur n'est pas égal à l'interpolation du module du champ aux nouds

Ces résultats ne sont pas surprenants (la distribution de nœuds suit le phénomène d'évanescence du champ). Ils permettent de valider la pertinence du critère [6] comme indicateur de l'erreur due au maillage.

\section{Conclusion}

Le critère énergétique présenté est novateur et permet une bonne appréciation de l'erreur dans les parties conductrices soumises à des courants induits. Il apparaît de plus que les éléments d'ordre 2 sont plus à même de rendre compte du couplage entre un élément et son entourage. Ainsi, en combinant le critère proposé et l'utilisation d'éléments d'ordre 2, on obtient une démarche originale, qui se démarque de l'évaluation classique d'une discontinuité numérique. On s'intéresse en effet à la conserva- 
tion intrinsèque de la puissance sur chaque élément, en utilisant le champ calculé sur l'élément lui-même pour évaluer l'erreur. Le cas $1 \mathrm{D}$ permet de valider cette démarche. Les travaux en cours consistent à mettre en place en $2 \mathrm{D}$ et $3 \mathrm{D}$ des procédures de remaillage à même de prendre en compte l'effet pelliculaire. Le caractère modulable et générique du critère permet de le coupler à n'importe quel remailleur. Une technique de remaillage utilisant le bubble meshing (Shimada et al., 1998), qui combine le déplacement et le rajout de nœuds, est à l'étude.

\section{Bibliographie}

Bossavit A., Computational electromagnetism: Variational formulations, complementarity, edge elements, vol. 3 of Electromagnetism, Academic Press, San Diego, USA, 1998.

Dufour S., Vinsard G., Laporte B., « Adaptation de maillage par modification de la position des noeuds ", NUMELEC'2000, Sessions Modèles Numériques pour l'Electromagnétisme, p. 26-27, 2000.

Dufour S., Vinsard G., Laporte B., « 2D adaptive mesh with movement », IEEE Transactions on Magnetics, vol. 37, n 5, p. 3482-3485, 2001.

Dufour S., Vinsard G., Laporte B., « Mesh improvement in 2-D eddy-current problems », IEEE Transaction on Magnetics, vol. 38, p. 377-380, 2002.

Jänicke L., Kost A., « Error estimation and adaptive mesh generation in the 2D and 3D finite element method », IEEE Transactions on Magnetics, vol. 32, n 3, p. 1334-1337, 2004.

Labridis D., « Comparative presentation of criteria for adaptative finite-element mesh generation in multiconductor eddy-current problems », IEEE Transaction on Magnetics, vol. $36, \mathrm{n}^{\circ} 1$, p. $267-280,2000$.

Li C., Ren Z., Razek A., « An approach to adaptive mesh refinement for three-dimensional eddycurrent computations », IEEE Transactions on Magnetics, vol. 30, n 1, p. 3482-3485, 1994.

Mazauric V., « Des principes thermodynamiques aux équations de Maxwell : Une approche variationnelle de l'électromagnétisme ", in G. Meunier (ed.), Champs et équations en électromagnétisme, vol. 1 of Electromagnétisme et éléments finis, Hermès, Paris, France, p. 147-262, 2003.

Mazauric V., "From thermostatistics to Maxwell's equations: A variational approach of electromagnetism », IEEE Transactions on Magnetics, vol. 40, n 2, p. 945-948, 2004.

Pinchuk A. R., Silvester P. P., « Error estimation for automatic adaptive finite element mesh », IEEE Transactions on Magnetics, vol. 21, $\mathrm{n}^{\circ}$ 6, p. 2551-2554, 1985.

Rikabi J., Bryant C., Freeman E., « An error-based approach to complementary formulations of static fields solutions », International Journal in Numerical Methods in Engineering, vol. 26, p. 1963-1987, 1988a.

Rikabi J., Bryant C., Freeman E., « Error-based derivation of complementary formulations for the eddy-current problem », IEE Proceedings, vol. 135, p. 208-216, 1988 b.

Shimada K., Gossard D., « Automatic triangular mesh generation of trimmed parametric surfaces for finite element analysis », Computer Aided Geometric Design, vol. 15, p. 199-222, 1998.

Sterz O., Hauser A.,Wittum G., « Adaptive local multigrid methods for solving timeharmonic eddy-currents problems ", IEEE Transaction on Magnetics, vol. 42, $\mathrm{n}^{\circ} 2$, p. 309-318, 2006. 
